\title{
Distributing Shared Regenerator Information in GMPLS-Controlled Translucent Networks
}

\author{
Nicola Sambo, Student Member, IEEE, Nicola Andriolli, Alessio Giorgetti, Filippo Cugini, \\ Luca Valcarenghi, Member, IEEE, and Piero Castoldi, Member, IEEE
}

\begin{abstract}
Sparse opto-electronic regenerators are used in optical networks to overcome optical signal quality degradations. Regenerators can be time-shared by dynamic lightpaths. However, current GMPLS control plane does not distribute regenerator information to network nodes.

Three regenerator information distribution methods for GMPLS-controlled networks are proposed. The first one, Regeneration Availability during Signaling (RAS), is based on the RSVPTE signaling protocol, while the others, Regeneration Capability Advertisement (RCA) and Regeneration Availability Advertisement $(R A A)$, are based on the OSPF-TE routing protocol.

Simulations show that RAA obtains the lowest blocking, while RCA the highest. Moreover, RAA only slightly increases the control plane load compared to RAS.
\end{abstract}

Index Terms-Translucent optical networks, regenerators, GMPLS, RSVP-TE, OSPF-TE.

\section{INTRODUCTION}

$\mathbf{I}$ $\mathrm{N}$ wavelength-routed optical networks (WRONs), the utilization of sparse (i.e., installed in a limited number of nodes) opto-electronic regenerators allows to overcome the reach limitations due to quality of transmission (QoT) degradation while keeping the network cost limited [1]. For this purpose, regenerators must be carefully placed during network planning [2]. In dynamic translucent WRONs ${ }^{1}$ a path for each incoming lightpath request must be found, specifying both link resources (i.e., wavelength channels) and node resources (i.e., opto-electronic regenerators) to be used. In addition, a lightpath source node must be provided with information about (i) link and node QoT parameters, and (ii) installed regenerators and/or regenerator availability in network nodes. Thanks to the former information, the lightpath source node becomes aware of the QoT degradation along every eligible path. Then, thanks to the latter one, in case of critical QoT, the source node can choose the regenerators to be exploited.

The GMPLS control plane is the natural candidate for disseminating this information in dynamic translucent WRONs. GMPLS is based on two main distributed protocols: the OSPFTE routing protocol and the RSVP-TE signaling protocol. OSPF-TE floods the network topology and traffic engineering

Manuscript received February 19, 2008. The associate editor coordinating the review of this letter and approving it for publication was N. Ghani. This work has been supported by MIUR under the Italy-Tunisia FIRB project "Software and Communication Platforms for High-Performance Collaborative Grid"' (RBIN043TKY).

N. Sambo, N. Andriolli, A. Giorgetti, L. Valcarenghi, and P. Castoldi are with Scuola Superiore Sant'Anna, Pisa, Italy (e-mail: nicola.sambo@sssup.it).

F. Cugini is with CNIT, Pisa, Italy.

Digital Object Identifier 10.1109/LCOMM.2008.080258.

${ }^{1}$ Dynamic translucent WRONs are optical networks with sparse shared regenerators in which lightpaths are dynamically set up. (e.g., bandwidth) information used for path computation, while RSVP-TE is responsible for the actual lightpath establishment.

However, no solutions have been standardized yet for the issue of encompassing QoT or regenerator information in GMPLS-controlled networks. To solve the former issue, [3] and [4] proposed to include QoT parameters into both RSVPTE and OSPF-TE protocols. This study focuses on the latter issue, thus it assumes that each node is aware of the relevant QoT parameters, so that each node can determine whether a path is critical in terms of QoT. Two solutions based on RSVPTE protocol extensions are proposed in [5] and [6] to include regenerator information into GMPLS protocol suite. In [5] and [6] regenerator information is gathered during the signaling procedure and stored at the source node, concerning the whole signaled path or each node along the path, respectively.

This paper proposes three novel solutions for distributing shared regenerator information. The solutions may be applied to both shared-per-node and shared-per-link regenerator architectures, using per-node or per-link regenerator information, respectively. While in [5] and [6], the gathered information is utilized by the source node only for the set up of a specific lightpath, in this paper, it is distributed to all network nodes and exploited also for subsequent lightpath requests. The first method, called Regeneration Availability during Signaling $(R A S)$, is based on RSVP-TE and exploits the extension proposed in [6] to distribute regenerator information carried by RSVP-TE messages to all traversed nodes. The other two methods, called Regeneration Capability Advertisement (RCA) and Regeneration Availability Advertisement (RAA), are based on OSPF-TE. While RCA advertises only the number of installed regenerators at each node, RAA advertises the number of regenerators that are currently available at each node. The three methods are compared in terms of blocking probability and control plane load. Simulation results show that: RAA minimizes the blocking probability at the expense of a slight increase of the control plane load; RAS minimizes the control plane load while achieving intermediate blocking probability; RCA achieves the worst performance in terms of blocking probability with a control plane load similar to RAA.

\section{SHARED REGENERATOR INFORMATION DisTRIBUTION METHODS}

The considered translucent network comprises $N$ nodes and $L$ bi-directional links. Each link supports $W$ wavelengths per direction. Each node consists of a photonic cross connect (PXC). $M \leq N$ nodes are equipped with a regeneration module composed of $r$ shared-per-node regenerators. The $M$ 
nodes are chosen with the signal quality prediction based regenerator placement algorithm (SQP) [7].

As proposed in [8], at each node the GMPLS control plane stores wavelength availability information in the traffic engineering database (TED), which is updated through OSPFTE flooding. Moreover, each node maintains an updated knowledge of the QoT parameters characterizing the optical layer [6], stored in a $Q o T$ parameter database (QPD). Therefore, each node can determine if a path is feasible or not in terms of resource availability and QoT.

When a lightpath request arrives at source node $s$ towards destination node $d, s$ computes a path through the Constraint Shortest Path First (CSPF) algorithm. Then, $s$ checks the QoT of the path by consulting QPD. If no transparent path is available, due to either resource unavailability or unacceptable QoT, the lightpath can be established only by using regenerators at intermediate nodes. It is assumed that each network node maintains a regenerator database $(R D)$ storing regenerator information (i.e., the number of installed and/or available regenerators at each node). $s$ designates nodes for regeneration based on the QoT information stored in its QPD and on the distributed regenerator information stored in its RD. The designated nodes are selected in order to partition the path into the minimum number of transparent segments with acceptable QoT. Note that more than one solution with the same number of designated nodes is possible. In this case the closest nodes to $s$ satisfying the above condition are designated.

Then, a signaling session starts from node $s$. The regenerator object (RO) [6] is included in the RSVP-TE messages. RO contains an entry for each node designated for the optoelectronic regeneration. When an intermediate node $i$ receives the RSVP-TE Path message, it checks if it is listed in RO. If so, it checks the availability of its regenerators. If no regenerators are available, it replaces the content of its RO entry with the downstream neighbor (thus handing the regeneration task to the next node along the path) and forwards the Path message. If node $i$ has at least one available regenerator, it consults QPD to check the QoT of the transparent segment terminating at $i$ (e.g., the fraction of the path from $s$ or the closest upstream regeneration node to $i$ ). If the segment has an acceptable QoT, node $i$ forwards the Path message without modifying RO, otherwise it sends a PathErr message to the source node. When the Path message reaches $d$, it verifies the QoT of the last transparent segment. If the path is admitted, $d$ sends a Resv message toward $s$ carrying a copy of the received $\mathrm{RO}$, otherwise it returns a PathErr message. Each intermediate node $i$ receiving the Resv message checks RO; if it is listed in $\mathrm{RO}$, it reserves a regenerator. When the Resv message reaches $s$ the lightpath is established, exploiting regenerators at the nodes listed in RO.

\section{A. Regeneration Availability during Signaling (RAS)}

In RAS, RSVP-TE messages are further extended with the Available Regenerator Object (ARO) [6]. Differently from [6], ARO is exploited at each node for updating RD with regenerator availability information of other nodes along the signaled path. During the signaling session, each node traversed by the Path message appends the number of its available regenerators to ARO. If the set up fails, ARO is copied into the PathErr message. At destination, if the path is admitted, ARO object is copied into the Resv message. In this way, each node traversed by RSVP-TE messages can refresh its RD with the updated regenerator availability information stored in ARO. Thus, when a source node requires a regeneration, it selects the path maximizing the number of currently available regenerators in the traversed nodes.

\section{B. Regeneration Capability Advertisement (RCA)}

Following [9], where additional WRON node information (e.g., wavelength conversion and regeneration capabilities) to be conveyed by the OSPF-TE routing protocol have been proposed, we define a novel regenerator information node state advertisement ( $R I-N S A)$ to distribute regenerator-related information. In particular the RCA method uses RI-NSAs to advertise the number of regenerators installed at each node. In RCA the RI-NSA flooding is triggered periodically. Every $T$ time units, each node creates an RI-NSA where it specifies its node-ID and the number of installed regenerators. Then, the RI-NSA is flooded in the network. Each node receiving the RI-NSA updates RD with the number of regenerators installed at the node indicated in the RI-NSA. Thus, when a source node requires a regeneration, it selects the path maximizing the number of installed regenerators in the traversed nodes.

\section{Regeneration Availability Advertisement (RAA)}

RAA uses RI-NSAs to advertise the number of currently available regenerators at each node. Besides the periodic triggering, in RAA RI-NSAs are flooded every time a regenerator is either reserved or released. The node creates an RI-NSA specifying its node-ID and the number of currently available regenerators. Then, the RI-NSA is flooded in the network. Each node receiving the RI-NSA updates the proper RD entry. Thus, when a source node requires a regeneration, it selects the path maximizing the number of currently available regenerators in the traversed nodes.

\section{Simulation RESUlTS}

The comparison among RAS, RCA and RAA is carried out by means of a custom-built $\mathrm{C}++$ event-driven simulator on the Pan-European network topology with 27 nodes and 55 links depicted in Fig. 1. Each link carries $W=40$ wavelengths per direction. $M=7$ nodes are equipped with a module of $r=8$ regenerators placed according to the SQP algorithm [7]. Lightpath requests are dynamically generated following a Poisson process with uniform distribution among the source-destination pairs. Both inter-arrival and holding times are exponentially distributed with an average of $1 / \lambda$ and $1 / \mu$, respectively. The load offered to the network is therefore expressed in Erlang as the ratio $\lambda / \mu$. The mean holding time is fixed to $1 / \mu=10^{4} \mathrm{~s}$.

For each lightpath request, CSPF computation is performed at source node $s$ considering all paths whose hop length is within one hop from the shortest path. If multiple equal cost shortest paths are available, a random choice is performed. If no path satisfies the QoT requirements, by consulting 


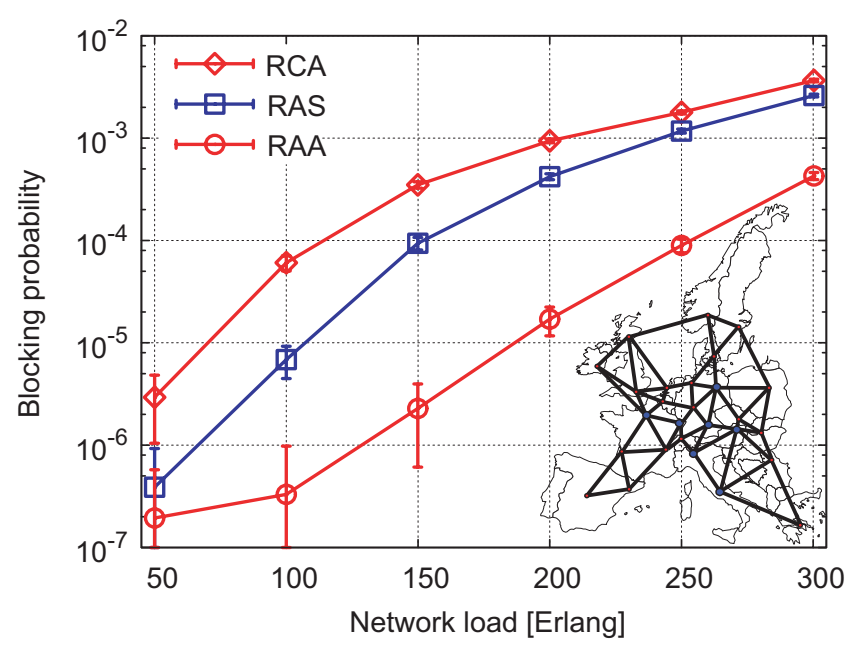

Fig. 1. Blocking probability vs network load for $1 / \mu=10^{4} s$.

QPD and $\mathrm{RD}$, the source node selects a path exploiting regenerators and fills $\mathrm{RO}$ as explained in Sec. II. A single set up attempt is performed for each lightpath request. The RI-NSAs generation period is set to $T=30$ minutes. All simulation results are plotted with the confidence interval at $95 \%$ confidence level.

Fig. 1 shows the blocking probability, expressed as the ratio between the number of blocked lightpaths and the number of lightpath requests, as a function of network load. The blocking probability increases with increasing network load for all the considered methods because regenerators are more frequently utilized and become scarce. RAS obtains better performance than RCA thanks to the utilization of regenerator availability information gathered by the RSVP-TE messages along traversed paths. However, both methods perform similarly for high network loads because frequent inter-arrivals make the availability information gathered in RAS quickly out-ofdate. RAA obtains the best performance because each time the status of a regenerator changes, the updated number of available regenerators is advertised to all network nodes.

Fig. 2 shows the control plane load, measured in OSPFTE regenerator information node and link state advertisement (RI-NSA and LSA) sent per second, as a function of network load. The considered LSAs are utilized for the advertisement of wavelength availability information as in [8]. RAS obtains the lightest control plane load. RCA and RAA present a slightly higher control plane load than RAS due to the utilization of the OSPF-TE protocol for advertising regenerator information. Moreover, the RAA control plane load is the highest because, unlike RCA, it triggers an RI-NSA packet each time the status of a regenerator changes. However, since for low network loads the regenerator status seldom changes, RCA and RAA require a similar number of NSAs. On the contrary, the difference between RCA and RAA control plane load is more evident for high network load, when frequent inter-arrivals lead to more frequent regenerator status changes.

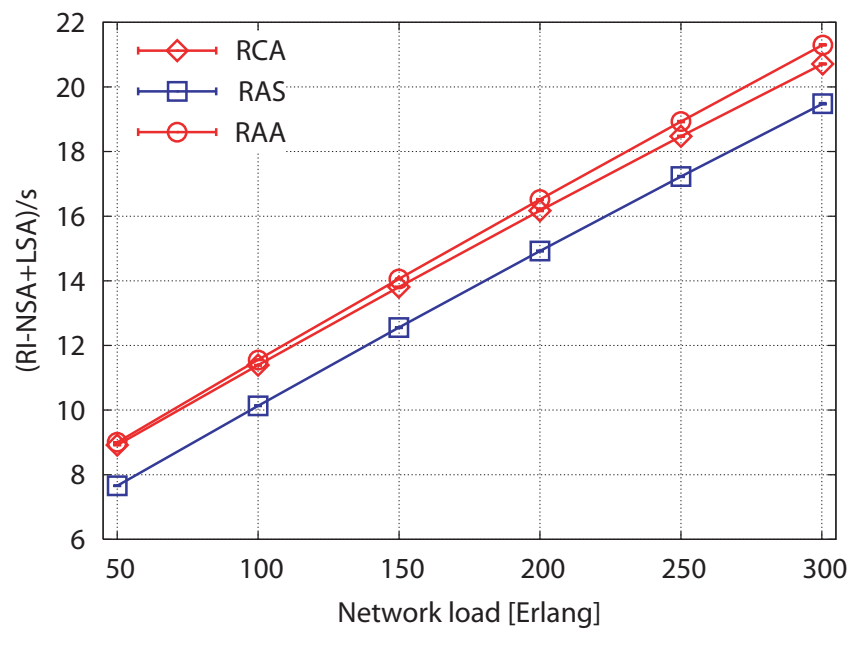

Fig. 2. Control plane load vs network load for $1 / \mu=10^{4} s$.

is based on RSVP-TE signaling protocol while both RCA and RAA methods are based on OSPF-TE routing protocol. Simulation results showed the benefit of distributing information about the number of currently available regenerators (as in RAS and RAA) instead of the number of installed regenerators (as in RCA). In the considered scenario, the RAS method outperforms the RCA method in terms of blocking probability, while minimizing the control plane load. On the other hand, the RAA method, advertising regenerator availability information, minimizes the blocking probability without considerably increasing the control plane load.

\section{REFERENCES}

[1] X. Yang and B. Ramamurthy, "Dynamic routing in translucent WDM optical networks: the intradomain case," J. Lightwave Technol., vol. 23, no. 3, pp. 1713-1723, Mar. 2005.

[2] S. Gangxiang and R. Tucker, "Translucent optical networks: the way forward," IEEE Commun. Mag., vol. 45, no. 2, pp. 48-54, Feb. 2007.

[3] R. Martinez, C. Pinart, F. Cugini, N. Andriolli, L. Valcarenghi, P. Castoldi, L. Wosinska, J. Comellas, and G. Junyent, "Challenges and requirements for introducing impairment-awareness into the management and control planes of ASON/GMPLS WDM networks," IEEE Commun. Mag., vol. 44 , no. 12 , pp. $76-85$, Dec. 2006.

[4] M. Ali, D. Elie-Dit-Cosaque, and L. Tancevski, "Enhancements to multiprotocol lambda switch to accommodate transmission impairments," in Proc. IEEE GLOBECOM 2001, Dec. 2001.

[5] N. Sambo, F. Cugini, N. Andriolli, A. Giorgetti, L. Valcarenghi, and P. Castoldi, "Lightweight RSVP-TE extensions to account for shared regenerators in translucent optical networks," in Proc. PIS '07, 2007.

[6] F. Cugini, N. Sambo, N. Andriolli, A. Giorgetti, L. Valcarenghi, P. Castoldi, E. L. Rouzic, and J. Poirrier, "GMPLS extensions to encompass shared regenerators in transparent optical networks," in Proc. ECOC 2007, Sept. 2007.

[7] X. Yang and B. Ramamurthy, "Sparse regeneration in translucent wavelength-routed optical networks: architecture, network design and wavelength routing," Photonic Network Commun., 2005.

[8] D. Li, Y. Lee, and J. Gao, "Evaluation of IGP extensions for wavelength switching optical networks," IETF Internet Draft, draft-li-ccamp-wsonigp-eval-00.txt, Dec. 2007, Internet draft.

[9] G. Bernstein, Y. Lee, and W. Imajuku, "Framework for GMPLS and PCE control of wavelength switched optical networks," draft-bernstein-ccampwavelength-switched-02.txt, Oct. 2007, Internet draft.

\section{CONCLUSION}

The paper presented three methods for distributing shared regenerator information in translucent networks. RAS method 